\title{
HIGHLIGHTS
}

OSTEOARTHRITIS

\section{A single genetic factor determines propensity to report musculoskeletal pain at multiple sites}

In conditions such as osteoarthritis $(\mathrm{OA})$, it is unclear whether reported musculoskeletal pain at different sites is related to pathological processes specific to individual joints or whether it reflects a more generalized tendency to experience pain. In a study of twins, more than $95 \%$ of the variance in patterns of painreporting across multiple anatomical sites could be explained by a single genetic 'common pain factor'.

"Clinical pain is one is the most important manifestations of OA," notes lead researcher Alex MacGregor, "and increasingly pain is used as a surrogate for radiographic OA in case-finding for studies attempting to achieve the large samples sizes required for genetic association analysis." However, the lack of correlation between clinical pain and radiographic disease in population studies of OA is well-known. The results of this study support the argument that the two should not be used as substitutes for each other in genetic studies of OA.

In the study, 991 pairs of monozygotic twins and 1,074 pairs of dizygotic twins from the TwinsUK cohort responded to a questionnaire in which they were asked whether they had experienced pain at the elbow, knee, thigh, hand, foot or neck and back in the previous month. The reported prevalence of pain at these sites ranged from $17 \%$ to $46 \%$, with a genetic influence suggested by the higher casewise concordance among monozygotic in comparison with dizygotic twins.

"Our analysis showed that the reporting of pain at different sites was uniformly correlated, and that most of the variation could be explained by a single common factor," reports MacGregor. "This factor was found to be strongly genetically determined, with a heritability of $46 \%$."

The findings sit in contrast to the group's previous work, which demonstrated that the genetic determination of radiographic OA is sitespecific. The data are, however, consistent with emerging evidence of a heritable basis for fibromyalgia and chronic widespread pain, conditions in which pain is experienced at multiple sites.

The investigators reason that the genetic basis of pain reporting and of radiographic change in OA are probably distinct. In addition, according to MacGregor, "since pain is of greater clinical importance than radiographic change to individuals with $\mathrm{OA}$, pursuing the genetic basis of pain reporting might be more relevant to those with the disease."

\section{Sarah Price}

Original article Williams, F. M. K. et al. Pain reporting at different body sites is explained by a single underlying genetic factor. Rheumatology (Oxford) doi:10.1093/ rheumatology/keq170 